International Journal of Engineering \& Technology, 7 (4.6) (2018) 103-107
International Journal of Engineering \& Technology
SPC
Website: www.sciencepubco.com/index.php/IJET
Research paper

\title{
Attribute Based Image Retrieval and Segmentation using On- tological Approaches
}

\author{
Ch. Mamatha ${ }^{1 *}$, Dr. V. Anandam ${ }^{2}$, Priyadarshini Chatterjee3, Hepshiba Vijaya Kumari ${ }^{4}$ \\ ${ }^{1}$ Department of Information Faculty, Vardhaman College of Engineering, Hyderabad \\ ${ }^{2}$ Department of Computer Science and Engineering, Vardhaman College of Engineering, Hyderabad \\ ${ }^{3}$ Department of Computer Science and Engineering, Vardhaman College of Engineering, Hyderabad \\ ${ }^{4}$ Department of Computer Science and Engineering MLR Institute of Technology, Hyderabad \\ *Corresponding author E-mail:mittumamatha03@gmail.com
}

\begin{abstract}
:
Content based image retrieval is gaining more and more importance as it is an apt approach to retrieve an image. The image is retrieved based on certain texture. Ontology is a branch of Meta Physics that helps in analyzing an input image based on certain textures. Ontology helps to retrieve an image based on its properties. Ontology describes a domain. With that domain, we can proceed further to understand the relation between the features present in the domain. There are biological-ontologies to analyze biological outcomes. The field of information technology can be combined with biological ontology to study the results of different biological effects. With the systematic concept of ontology that includes rules, classes, relations etc we can understand an image better that eventually helps in accurate image retrieval. Ontology can be generic or domain specific. In this paper we will be using domain specific ontology used to analyze the features of digital images along with image segmentation to retrieve an image. We will be testing our proposed system using the colored images of mammals. In case of image segmentation we will using the general techniques already existing.
\end{abstract}

Keywords: Attribute based image retrieval, Ontology, Image segmentation.

\section{Introduction:}

In recent years, the evolution of information has become more outstanding in order to retrieve information or data in huge assortment to provide fastest access and trading all over the world. In distributed environments we make use of structured kind of representation which adopts the concept of ontology with process semantic representation of a domain. Ontology can be used in transferring and processing data or information in an effective manner. We can use many methodologies in this process, which involves in extracting various concepts and semantic relations from the respective unstructured assets.

As there is huge increase in demand for digital images along with evolution of WWW (World Wide Web). The exploration of processing the desired image in a huge assortment is very big task. Photography plays a vital role in facilitating the communication and capturing of digital images.

The most significant inventions which related to web services application which includes self describing and self contained which automatically share information among various applications over internet. The ground work towards the concept of similarity extraction come with vocabularies along with communication which builds a meaning in web based applications. The need in finding related images or desired one from a huge collection of images will play a very important role in many professions. Where their requirements may differ from one another based the queries so far which includes in characterizing the images using feature extraction which may consider color, shape, objects and there logical features based on demand levels of the user. Primary features in characterization of image may include shape color both for query and as well stored images which attempts in retrieving.

Content based image retrieval includes processing, searching and finding the query image in an effective manner. Where the images can be segmented and retrieved based on color shape and texture of the query image which we have. In this process of identification user tends to select a region of image and system may return images based on the region from large collection of images which may take many feature extraction for processing as discussed which may take many considerations based on need and user demand.

The theory of image extraction and image segmentation is an important in processing in pattern recognition and image pre processing. The aim which can be achieved using image segmentation which used in simplifying the representation of image in analyzing and providing meaningful extraction using locating objects.

Ontology involves in relating the information which includes machine interpretable features within the domain and the relations among them. There can be plenty of reasons in developing an ontology which also includes explicit descriptions within the domain of discourse with knowledge base. In order to extract information automatically using textual content it integrating different levels of different types of knowledge base includes syntactic, lexical and semantic and usually pragmatic. Ontology can be applied in many of these concepts to extract raw information. 
Ontology methodology deals with methodologies and process of assets along with the issues which is used to provide guidelines in advancing ontology for researchers. Theoretical approaches allows us in describing the most general features in an abstract way with tend to provide better understanding as well in maintaining the data with match in implementing applications.

\section{Related Work:}

Image retrieval plays a vital role in pattern recognition and artificial intelligence areas which may include many categories like content based, semantic based and text based in processing and retrieving images. The query image can be taken as sample in extracting the similarity among huge assortment of images in order to retrieve desired image among them. The process of extraction needs an accurate segmentation method in image which points the similarities among regions or boundaries [1] The consideration can be of many kinds one of among them can deal with edge based representation for image retrieval which is introduced with the concept of edge direction histogram for trade marking. This method is well balanced with image rotation and scaling as it concentrates on edges and translation [2] [3] Many features can be considered like shape and texture using multi scale along with edge considerations in image retrieval. Color, shape and texture are fundamental considerations which are used in image segmentation which is more useful in pattern recognition [4]. Content based retrieval can be classified as 3 categories in which we can retrieve image based on primary features like color, shape and texture. Whereas other retrieving based on objects using logical inference and last by abstract attributes which may include significant pictures based on high level reasoning [5].

The problem of attaining based on pattern recognition, automatic detection, image segmentation based on objects or regions which should be done in meaningful manner. Not only reliability of segmentation and pattern recognition is a challenge in recognizing both structured and unstructured data in large amount but also the efficiency of algorithm will plays a vital role while dealing with huge amount of data [6]. Object detection can be done by focusing on edge fragments as a group in the image based on local shaping using part bundles which make use of overlapping fragments as a group [7].

Cortina provides image retrieval system using image search in millions of images using text annotations and image content. It collects information in web crawler using information based on category, keywords along with content descriptors. It also provides the feature of identifying duplicate images in the huge assortment of database with consist of millions of images. The systems include image acquisition, clustering and indexing and also feature extraction [8].

Images are the great source of content over internet. Image retrieval and analysis acts is important for recent trends in image processing research. Attribute based image retrieval which is decision based approach which provides retrieves content based on keywords or text or images itself. Attribute image retrieval which uses color, shape and texture based classification [9] Single feature extraction will be unsatisfactory in content based retrieval, the system can come across with two or more feature extraction at a time suing image color, image shape and image texture which describes image content based on representation[10].

Ontology uses common components in content based retrieval which includes many things relationships, attributes, classes and functions etc. ontology based implementation deals with hierarchical representation between concepts and their relationships [11][12][13]. The feature extraction between classes and relationships can be namely part of or instance of based on ontology which come up with sharing common features among entities[14][15].
In semantic web the resultant or information of retrieved content for user query should be in well balanced and in meaningful structure. Where to existing data documents it can acts as metadata which extends into well defined information for query. It enables the extension for searching or processing related information automatically by user as well by machines [16].

The conventional method for retrieval of digital images in semantic web investigates using image based analysis using logical features or attributes or either by image retrieval based on similarities [17][18]. Now a day's image retrieval is done by text-based or keyword based which is still in lack of providing meaningful information among many search engines [19] [20]. The traditional goal of ontology is to relate real world objects in very absolute way in order to divide the word in order which helps in discovering in which the objects may really fall[21][22]. Ontology plays a vital role in knowledge based system which is a basic step in constructing domain ontology [23].

The principles involves in similarity matching of images automatically from the database retrieval which indicates many difficulty in generating similarity based search [24]. The framework used in retrieving images can include classification, in which query image can be considered in series of levels into at which it meets the user requirements in extracting features [25]. Content based image retrieval explains the principals which deal with extraction based on color, shape, spatial indexing and texture can be considered in image segmentation to provide solutions for the issues in retrieving and comparing compressed images [26].

Digital image retrieval and processing is one of the major important research field from past year in which propose many approaches or methods in analyzing image properties [27] [28]. As discussed earlier retrieval of images can be done or searching can take place by making use of key-words, Meta data or text. Where the information related to query image is given by user which can be searched using many search engines.

Image based input query which creates query image as input based on the query image certain searching techniques are performed. Based on the objects detected certain steps are taken place in order to perform image segmentation. First step involves ion extracting similarities based on texture and shape and which followed by color value and pixel color detection. Second texture classification takes place in order to detect similarity percentage among detected images [29].

\section{Proposed System:}

Our proposed approach will be divided into three parts:

1. Acquiring a digital image

2. Feature extraction of the acquired image using content based image retrieval

3. Applying Ontological concepts to retrieve the image

4. Applying image segmentation techniques to segment the image in order to highlight important parts of the image

First step we need to do is to acquire the image from the image database. In order to acquire an image from the database, we should be clear of the purpose of retrieving the image. We need to form certain queries to get the images from the image database. These queries will be related to the kind of images that we want to retrieve. Of course, the kind of images will be related to the application we are trying to design. Foremost the query should be able to answer why the users want to retrieve the images. What will be the uses of the images in the application that the user intend to design? May be image is required illustrate any kind of text articles, required to convey the emotion of any particular word in the text document. The image may be also required as a detailed description of any specific domain related problem to be solved.The image that we retrieve might depict a particular type of object, might convey a specific pattern, a typical texture, or a specific mood. The image can be retrieved 
based on its certain type of attributes like texture, color, shape, or possessing certain arrangement of objects, or based on certain patterns present in the image. The proposed paper deals with retrieval of the image based on shape and color properties. In the proposed system we will be designing the query motor that will take input of any digital image of a mammal. Inside the query engine, there is a query matching segment that will consult with the knowledge repository to search the image from the database. The knowledge repository is trained by the ontological concepts. Theses ontological concepts will be provided by a segment called the 'ontology governor'. There is ranking segment that is going to grade the image.

After the retrieval of the image, the last part of the proposed system is to segment the image in similar part. We will adhere to edge based image segmentation. Image segmentation is an ideal step to be applied after content based image retrieval. Describing each part of the proposed system briefly.

1. The query motor: The query motor takes input from the user using online interfaces. The input carries the content the user needs to search. The input can be in the form of images or texts. The proposed system takes image input. The image input can contain multiple or single objects. This approach is used as the search of the user will be specific to the image that he needs for the application. The query motor will be using image related query. The question asked will be regarding the shape of the object. After the answer of the first question, the next question that is asked is on the color of the image. Here we need to find the color value of the pixels using hue, saturation and value model. After the second step, we need to find the texture of the image. At the fourth step, the lower level parameters obtained in the previous step, is converted to higher level parameters using ontological concepts. We will generating a SPARQL query.

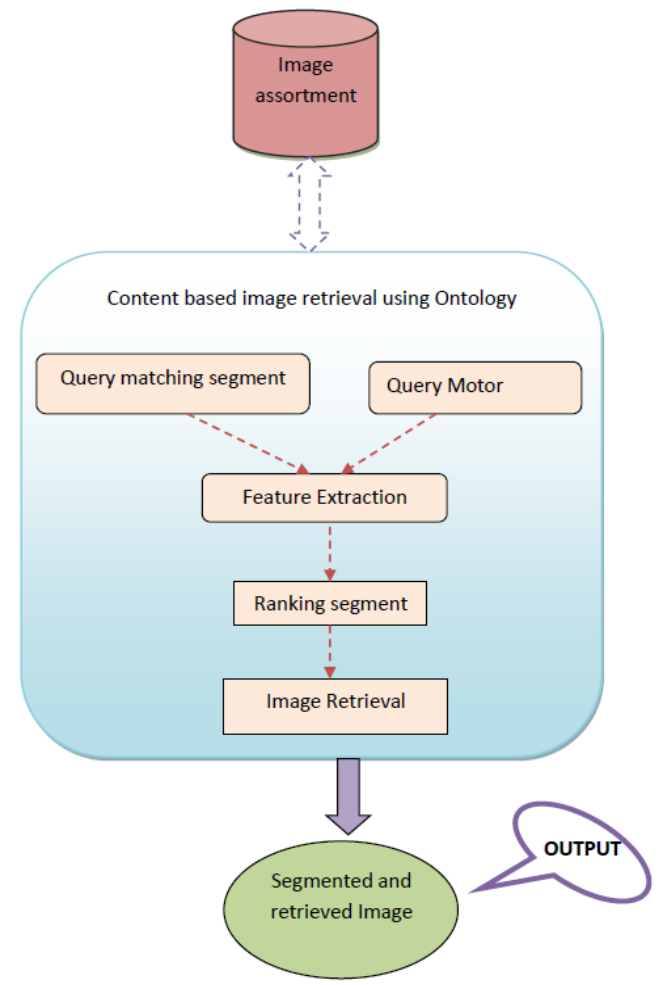

Figure 1: Attribute Based Image retrieval and Segmentation using Ontological Approaches

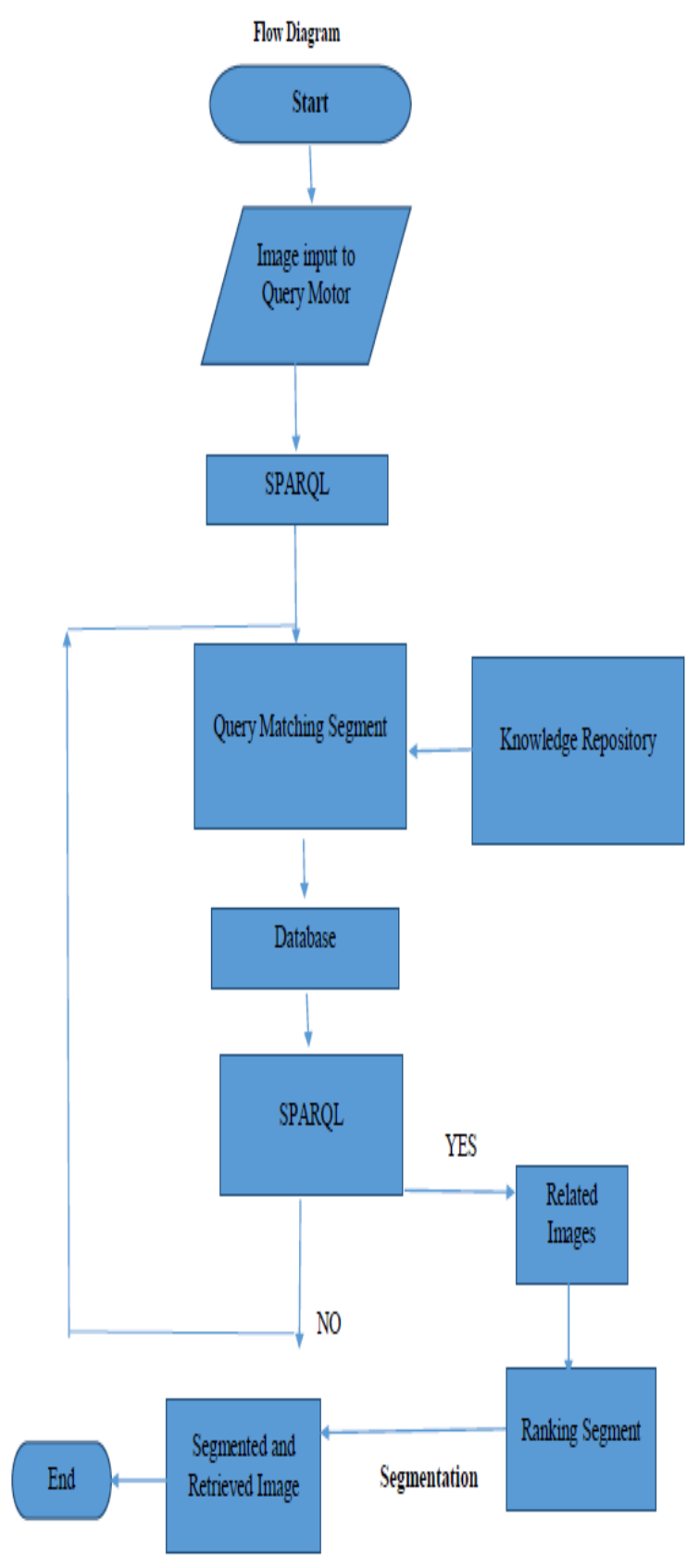

Figure 2: flow diagram of the system

2. Query matching segment: The query matching segment is going to take the sparkle query as input from the query motor. With the help of knowledge repository that is based on ontological concepts is able to find related images from the database.

3. Ranking segment: if the query is successfully executed, then result of the query is passed for grading. If the results are unsuccessful, the matching segment again search the image database. The ranking segment will provide ranks to images analyzing the need of the users. The result set is stored in ascending order relative to the rank.

4. Image processing segment. After ranking the image, the ranked images from high to low order are passed to the image processing segment that will partition the images using edge based image segmentation algorithms.

In the HSV color space we consider these three parameters to segment the image. Hue is the angle that is directly related with the color. Saturation is with respect to the purity of the color. The value part represents percentage of value from 0 to 100 . Value also says the amount of light by which a color is illumi- 
nated. We will be using this algorithm to partition the image in our proposed system.

The ontology governor is trained with the ontological concepts using 90 images of 5 different mammals.

\section{Result Analysis:}

As said in the proposedsystem, we will be testing our system with 90 images of 5 different mammals. The system will be evaluated on the basis of test cases. The results were quiet good and comprehensible.

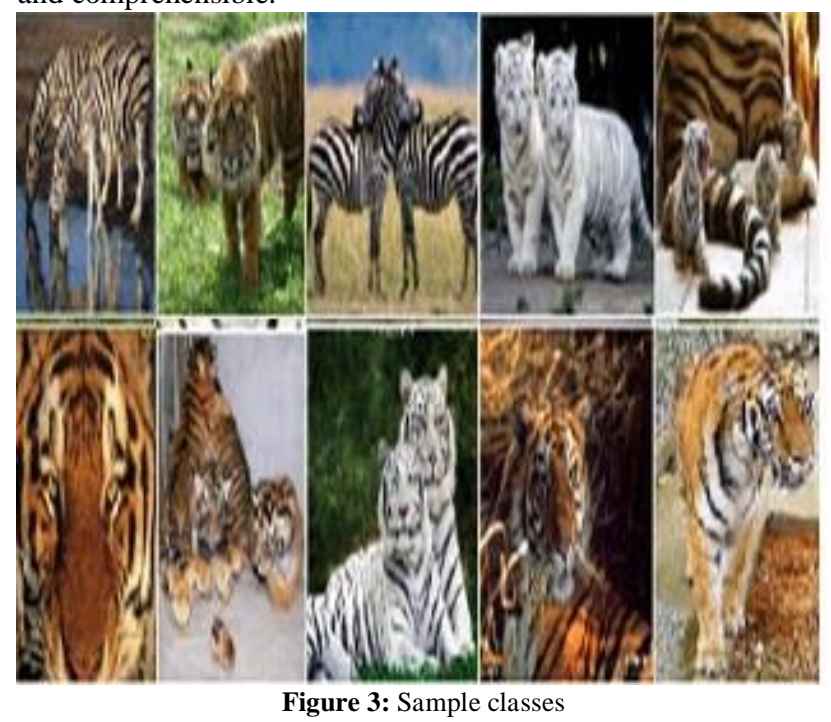

Test Cases Against Correctness

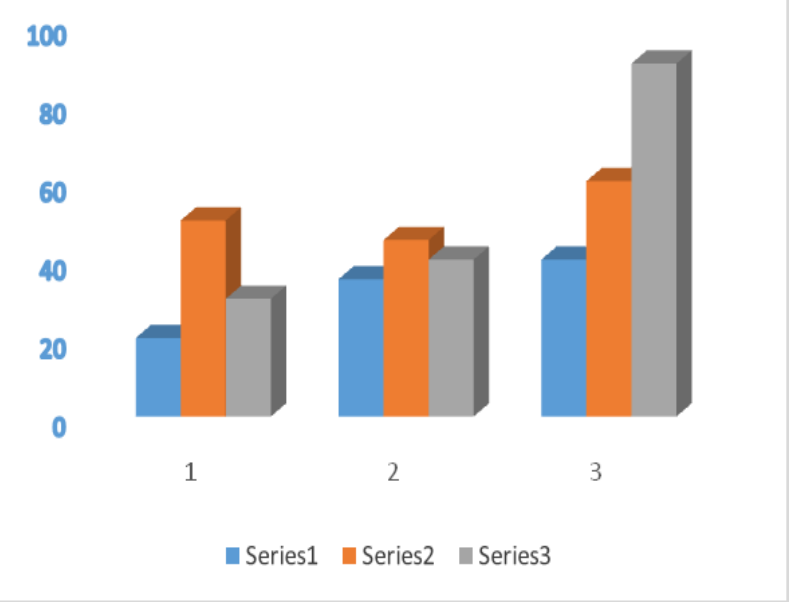

Figure: 4 test cases

As per figure 5, the blue line shows the result of our approach. The orange and the grey line shows the result of other image retrieval approaches.

Figure 4 shows the accuracy rate of our proposed system, as compared to the other systems present. Figure 5 depicts the percentage improvement chart of our proposed system as compared with other systems.

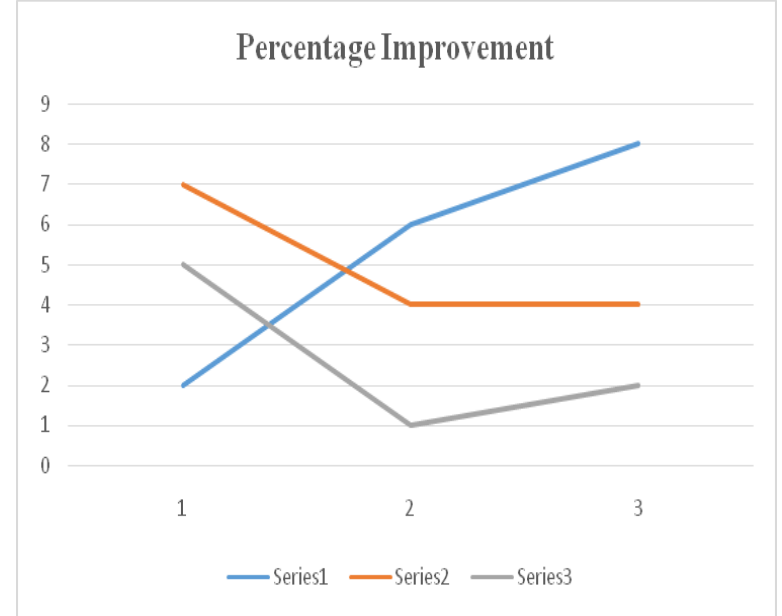

Figure 5: Percentage Improvement

\section{Conclusion:}

There are various approaches to image retrieval system that is primarily based on Meta data. The common search engines searches based on keywords. Though this approach is fast, it has some negative outcomes. The attribute based image retrieval techniques retrieves the images based on comparing the features of the images. There are various researches carried in the past on ABIR. There are some negative outcomes of ABIR also like ABIR fails to clearly understand the meaning of images. For this reason, our proposed system uses Ontological concepts to help the search engine, to understand the meaning of images and then find a related set of images based on the SPARQL. This SPARQL is generated by the query motor, taking into consideration various features of the input images.

The knowledge repository is trained with ontological concepts that helps to translate the low level image features to high level features. This step helps in better understanding the semantics of the image. Above graphs clearly shows how our method has outperformed other approaches to image retrieval techniques along with segmentation.

\section{References:}

[1] Guang-Hai Liu, Lei Zhang, Ying-Kun Hou, Zuo-Yong Li, Jing-Yu Yang, Image retrieval based on multi-texton histogram, Pattern Recognition (2010), Volume 43, Pages 2380-2389.

[2] A.K. Jain, A. Vailaya, Image retrieval using color and shape, Pattern Recognition 29 (8) (1996) 1233-1244.

[3] A.K. Jain, A. Vailaya, Shape-based retrieval: a case study with trademark image database, Pattern Recognition 31 (9) (1998) 1369-1390.

[4] S. Kiranyaz, M. Ferreira, M. Gabbouj, A generic shape/texture descriptor over multiscale edge field: 2-D walking ant histogram, IEEE Transactions on Image processing 17 (3) (2008) 377-390.

[5] Y. Liu,D. Zhang,G. Lu,W.-Y. Ma,A survey of content-based image retrieval with high-level semantics, Pattern Recognition (2007), Volume 40, Issue 11, Pages 262-282.

[6] Shotton J, Winn J, Rother C, Criminisi A (2009) Texton boost for image understanding: Multi-class object recognition and segmentation by jointly modeling texture, layout, and context. International Journal of Computer Vision, 81(1), 2009.

[7] Shotton J, Blake A, Chipolla R, Contour-based learning for object detection. In Proceeding of International Conference on Computer Vision (2005)

[8] T. Quack, U. Monich,L. Thiele, B.S. Manjunath, Cortina: a system for large-scale, content-based web image retrieval, in:Proceedings of the 12th annual ACM international conference on Multimedia, 2004.

[9] Umar Manzoor, NaveedEjaz, Nadeem Akhtar, Muhammad Umar, M Shoaib Khan, Hafsa Umar "Ontology based image retrieval", 
IEEE The 7th International Conference for Internet Technology And Secured Transactions (ICITST-2012), pp. 288-293, 2012.

[10] Ming Zhang, Ke Zhang, Qinghe Feng, Jianzhong Wang, Jun Kong, Yinghua Lu "A novel image retrieval method based on hybrid information descriptors" Journal of Visual Communication and Image Representation, Volume 25, Issue 7, October 2014, Pages 1574-1587.

[11] Umar Manzoor, Samia Nefti, Yacine Rezgui, —Categorization of malicious behaviors using ontology-based cognitive agentsl, Data \& Knowledge Engineering (2013), Volume 85, May 2013, Pages 40-56.

[12] Umar Manzoor, Samia Nefti, —iDetect: Content Based Monitoring of Complex Networks using Mobile Agentsll, Applied Soft Computing, Volume 12, Issue 5, May 2012, Pages 1607-1619.

[13] Umar Manzoor, Samia Nefti, Yacine Rezgui "Autonomous Malicious Activity Inspector - AMAI" Natural Language Processing and Information Systems, Lecture Notes in Computer Science Volume 6177, 2010, pp 204-215.

[14] Francesco Rea, Samia Nefti-Meziani, Umar Manzoor, Steve Davis "Ontology enhancing process for a situated and curiositydriven robot" Robotics and Autonomous Systems, Volume 62, Issue 12, December 2014, Pages 1837-1847.

[15] Umar Manzoor, Mati Ullah, Arshad Ali, Janita Irfan, Muhammad Murtaza "A Tool for Agent Based Modeling - A Land Market Case Study" Information Systems, E-learning, and Knowledge Management Research, Communications in Computer and Information Science Volume 278, 2013, pp 467-472.

[16] Dacheng Tao, Dianhui Wang, FionnMurtagh, "Machine learning in intelligent image processing", Signal Processing, Volume 93, Issue 6, June 2013, Pages 1399-1400.

[17] T. Quack, U. Monich, L. Thiele, and B. S. Manjunath, "Cortina: A System for Large-Scale, Content- Based Web Image Retrieval", In Proceedings of ACM international conference on Multimedia, (2004).

[18]M. Zhang, K. Zhang, Q. Feng, J. Wang, J. Kong, and Y. Lu "A Novel Image Retrieval Method Based on Hybrid Information Descriptors", Journal of Visual Communication and Image Representation, vol. no. 257, (2014), pp. 1574-1587

[19] Y . Liu, D. Zhang, G. Lu, and W.-Y. Ma, "A Survey of ContentBased Image Retrieval with High-Level Semantics", In Pattern Recognition, vol. 40, no. 11, (2007), pp. 262-282.

[20] A. Al Azemi, S. Nefti, U. Manzoor, and Y. Rezgui, "Building a Bilingual Bio-Ontology Platform for Knowledge Discovery", International Journal of Innovative Computing, Information and Control, vol. 7, no. 12, (2011), pp. 7067-7075.

[21] R.-C. Chen, C.-T. Bau, M.-Y. Tsai and C.-Y. Huang, Web pages cluster based on the relations of mapping keywords to ontology concept hierarchy, International Journal of Innovative Computing, Information and Control, vol.6, no.6, pp.2749-2760, 2010.

[22] G. T. Raju, P. S. Satyanarayana and L. M. Patnaik, Knowledge discovery from web usage data: Extraction and applications of sequential and clustering patterns - A survey, International Journal of Innovative Computing, Information and Control, vol.4, no.2, pp.381-389, 2008.

[23] D. Jones, T. Bench-Caponand and P. Visser, Methodologies for ontology development, Proc. of IT \& KNOWS Conference of the 15th IFIP World Computer Congress, 1998.

[24] Aigrain, P et al (1996) "Content-based representation and retrieval of visual media - a state-of-the-art review" Multimedia Tools and Applications 3(3), 179-202.

[25] Eakins, J P (1996) "Automatic image content retrieval - are we getting anywhere?" Proceedings of Third International Conference on Electronic Library and Visual Information Research (ELVIRA3), De Montfort University, Milton Keynes, pp 123-135

[26] Idris F and Panchanathan, S (1997a) "Review of image and video indexing techniques" Journal of Visual Communication and Image Representation 8(2) 146-166.

[27] Shotton J, Winn J, Rother C, Criminisi A (2009) Texton boost for image understanding: Multi-class object recognition and segmentation by jointly modeling texture, layout, and context. International Journal of Computer Vision, 81(1), 2009.

[28] Shotton J, Blake A, Chipolla R, Contour-based learning for object detection. In Proceeding of International Conference on Computer Vision (2005)

[29] Guang-Hai Liu, Lei Zhang, Ying-Kun Hou, Zuo-Yong Li, JingYu Yang, Image retrieval based on multi-texton histogram, Pattern Recognition (2010), Volume 43, Pages 2380-2389. 